\title{
Adaptive Observers for Structurally Perturbed Positive Real Delay Systems
}

\author{
M. A. Demetriou* \\ R. F. Curtain ${ }^{\dagger}$ \\ K. Ito
}

\begin{abstract}
The aim of this investigation is to construct an adaptive observer for a class of multivariable delay systems having a known exogenous input and a structured perturbation with an unknown constant parameter, such as the case of static output feedback with an unknown gain. The adaptive observer uses the nominal dynamics of the unperturbed plant and an adaptation law based on the Lyapunov redesign method. We obtain conditions on the system to ensure uniform boundedness of the estimator dynamics and the parameter estimates, and convergence of the estimator error. We illustrate our approach with a multivariable delay example for which we obtain convincing numerical results.
\end{abstract}

\section{Introduction}

In Curtain, Demetriou and Ito [2] we constructed adaptive observers for the following class of infinite-dimensional systems on a given Hilbert space $X$ with inner product and norm $\langle\cdot, \cdot\rangle$ and $|\cdot|_{X}$, respectively:

$$
\begin{aligned}
& \frac{d}{d t} x(t)=A_{p} x(t)+B u(t)+f(t) ; \quad x(0)=x_{0} \in X \\
& y(t)=C x(t),
\end{aligned}
$$

where

$$
A_{p}=A_{0}+B \Gamma C
$$

and $A_{0}$ is a generator of an exponentially stable $C_{0}$-semigroup $T(t)$, i.e. there exists constants $M, \mu>0$ such that

$$
\|T(t)\| \leq M e^{-\mu t} .
$$

The signals $u(t)$ and $y(t)$ are the vector-valued inputs and outputs, respectively. $f(t)$ is an $X$-valued known exogenous input and $B \in \mathcal{L}\left(\mathbb{C}^{m}, X\right), C \in \mathcal{L}\left(X, \mathbb{C}^{m}\right)$. Consequently, $A_{p}$ generates a strongly continuous $C_{0}$-semigroup for any $\Gamma$ and the system is well-posed. A key assumption is that the original system $\left(A_{0}, B, C\right)$ satisfy a positive-real condition, where by positive-real we mean the following.

Definition 1.1 Suppose that $G(\cdot): \mathbb{C}_{0}^{+} \rightarrow \mathcal{L}\left(\mathbb{C}^{m}\right)$ where $\mathbb{C}_{0}^{+}=\{s:$ Res $>0\}$. If

$$
\begin{aligned}
& \text { (i) } \overline{G(s)}=G(\bar{s}) \\
& \text { (ii) } G(s) \text { is holomorphic on } \mathbb{C}_{0}^{+} \\
& \text {(iii) } G(s)+G(s)^{*} \geq 0 \text { for all } s=j \omega, \omega \in \mathbb{R},
\end{aligned}
$$

then $G$ is positive-real.

\footnotetext{
${ }^{*}$ Department of Mechanical Engineering, Worcester Polytechnic Institute, Worcester, MA 01609, USA, email: mdemetri@wpi.edu.

${ }^{\dagger}$ Department of Mathematics, University of Groningen, P.O. Box 800, 9700 AV Groningen, The Netherlands, email: r.f.curtain@math.rug.nl.

${ }^{\ddagger}$ Center for Research in Scientific Computation, North Carolina State University, Raleigh, NC 27695, USA, email: kito@eos.ncsu.edu. Research supported in part by the Airforce Office of Scientific Research under grant AFOSR-49620-95-1-0447.
} 
In fact, we require that $G(s-\mu)=C\left((s-\mu) I-A_{0}\right)^{-1} B$ be positive-real for some $\mu>0$.

The motivation is that the perturbation $B \Gamma C$ arises as the unknown constant gain in the feedback law $u=\Gamma y$. Thus, the actual formulation is

$$
\dot{x}(t)=A_{0} x(t)+B \Gamma y(t)+B u(t)+f(t) ; \quad x(0)=x_{0} .
$$

The proposed state estimator (observer) is

$$
\dot{\widehat{x}}(t)=A_{0} \widehat{x}(t)+B u(t)+B \widehat{\Gamma}(t) y(t)+f(t), \quad \widehat{x}(0)=\widehat{x}_{0},
$$

where $\widehat{x}(t)$ is the state estimate at time $t$ and $\widehat{\Gamma}(t)$ is the adaptive estimate of the unknown gain. The adaptation rule for $\widehat{\Gamma}(t)$ was based on the Lyapunov redesign method $[5,6]$ that has proved successful for finite-dimensional systems and it depends on the existence of a solution to a certain constrained Lyapunov equation. The following is the main result from Curtain, Demetriou and Ito [2].

Theorem 1.2 Consider the structurally perturbed system (1.1)-(1.3), where $A_{0}$ is the generator of an exponentially stable semigroup on $X, B \in \mathcal{L}\left(\mathbb{C}^{m}, X\right), C \in \mathcal{L}\left(X, \mathbb{C}^{m}\right), f(t)$ is a known exogenous signal with values in $X$, and $\Gamma$ is an unknown matrix feedback gain. If there exist $Q \in \mathcal{L}(X)$ and $L \in \mathcal{L}(D(A), X)$ or $\mathcal{L}\left(D(A), \mathbb{C}^{m}\right)$ satisfying the constrained Lyapunov equation for $x \in D(A)$

$$
\begin{aligned}
& \left(A_{0}+\mu I\right)^{*} Q x+Q\left(A_{0}+\mu I\right) x=-L^{*} L x \\
& B^{*} Q x=C x
\end{aligned}
$$

then the state estimator defined by (1.9) and the adaptation rule with adaptation matrix gain $G=G^{T}>0$ given by

$$
\begin{aligned}
& \dot{\hat{\Gamma}}(t)=G C e(t) y^{T}(t) \\
& \widehat{\Gamma}(0)=\widehat{\Gamma}_{0}
\end{aligned}
$$

have the following properties.

(i) If $u, y \in L_{\infty}\left(0, \infty ; \mathbb{C}^{m}\right)$, then $\widehat{\Gamma}(t), \widehat{x}(t)$ and the estimation error $e(t)=x(t)-\widehat{x}(t)$ are bounded in norm for $t \geq 0$ and $\left\|e^{\frac{\mu}{2} t} Q^{\frac{1}{2}}(x(t)-\widehat{x}(t))\right\|_{X} \rightarrow 0$ as $t \rightarrow \infty$.

(ii) If $y \in L_{\infty}\left(0, \infty ; \mathbb{C}^{m}\right) \cap L_{2}\left(0, \infty ; \mathbb{C}^{m}\right)$ then $\left\|e^{\frac{\mu}{2} t}(\widehat{x}(t)-x(t))\right\|_{X} \rightarrow 0$ as $t \rightarrow \infty$

(iii) If the conditions in (ii) hold and, in addition, the plant is persistently exciting, i.e. there exists $T_{0}, \delta_{0}$ and $\epsilon_{0}$ such that for each sufficiently large $t>0$ there exists $\tilde{t} \in\left[t, t+T_{0}\right]$ such that

$$
\left|\int_{\tilde{t}}^{\tilde{t}+\delta_{0}} y^{T}(\tau) \mathbf{w} d \tau\right| \geq \epsilon_{0}
$$

for every unit vector $\mathbf{w} \in \mathbb{R}^{m}$, then we can achieve parameter convergence, i.e.

$$
\widehat{\Gamma}(t) \rightarrow \Gamma, \quad \text { as } t \rightarrow \infty
$$

The crucial assumption, that the constrained Lyapunov equation (1.10)- (1.11) has a solution, is a difficult problem for infinite-dimensional systems and only partial results are available. In Curtain, Demetriou and Ito [2], we designed adaptive observers for scalar systems of parabolic and delay type by exploiting recent results on constrained Lyapunov equations in Curtain [1]. While the theory also applied to multivariable systems, the conditions prove to be difficult to verify in the examples. It is the aim of this paper to apply the theory to design an adaptive observer for a class of multivariable retarded systems. We demonstrate the power of the results by numerical simulations. 


\section{A class of positive-real retarded systems}

We consider the following class of retarded systems

$$
\begin{aligned}
& \dot{x}(t)=A_{0} x(t)+\alpha x(t-h)+B_{0} u(t) ; \\
& x(0)=r, x(\theta)=f(\theta),-h \leq \theta<0 ; \\
& y(t)=C_{0} x(t),
\end{aligned}
$$

where $h>0$ represents the point delay, $x(t) \in \mathbb{C}^{n}, A_{0} \in \mathcal{L}\left(\mathbb{C}^{n}\right), B_{0} \in \mathcal{L}\left(\mathbb{C}^{m}, \mathbb{C}^{n}\right), C_{0} \in$ $\mathcal{L}\left(\mathbb{C}^{n}, \mathbb{C}^{m}\right), r \in \mathbb{C}^{n}$, and $f \in \mathbf{L}_{2}\left(-h, 0 ; \mathbb{C}^{n}\right)$. The delay system (2.1)- (2.3) can be formulated on the state space $X=\mathbb{C}^{n} \oplus \mathbf{L}_{2}\left(-h, 0 ; \mathbb{C}^{n}\right)$ with generating operators defined by

$$
\begin{gathered}
B u=\left(\begin{array}{c}
B_{0} u \\
0
\end{array}\right), \quad C\left(\begin{array}{c}
r \\
f(\cdot)
\end{array}\right)=C_{0} r, \\
\mathcal{D}(A)=\left\{\begin{array}{c}
\left(\begin{array}{c}
r \\
f(\cdot)
\end{array}\right) \in X \mid f \text { is absolutely continuous, } \\
\frac{d f}{d \theta}(\cdot) \in \mathbf{L}_{2}\left(-h, 0 ; \mathbb{C}^{n}\right) \text { and } f(0)=r
\end{array}\right\}, \\
A\left(\begin{array}{c}
r \\
f(\cdot)
\end{array}\right)=\left(\begin{array}{c}
A_{0} r+\alpha f(-h) \\
\frac{d f}{d \theta}
\end{array}\right) .
\end{gathered}
$$

$A$ generates a $C_{0}$ semigroup $T(\cdot)$ on $X$ and $B, C$ are bounded operators (see Curtain and Zwart [3], chapter 2.4 and Theorems 4.2.6, 4.2.10). The spectrum of $A, \sigma(A)$ consists of eigenvalues which are the solutions of $\operatorname{det}(\Delta(\lambda))=0$, where for $\lambda \in \mathbb{C}$

$$
\Delta(\lambda)=\lambda I-A_{0}-\alpha e^{-\lambda h} .
$$

$T(\cdot)$ is exponentially stable if

$$
\sigma(A) \subset\{s \mid \operatorname{Re} s<-\mu\} \quad \text { for some } \mu>0 .
$$

The transfer function $G$ of the system is given by

$$
G(s)=C_{0} \Delta(s)^{-1} B_{0} .
$$

We also need to consider a more general type of observation operator

$$
C_{\Xi}\left(\begin{array}{c}
r \\
f(\cdot)
\end{array}\right)=C_{1} r+C_{2} f(-h),
$$

where $C_{j} \in \mathcal{L}\left(\mathbb{C}^{n}, \mathbb{C}^{q}\right), j=1,2$. The transfer function $\Xi$ of the system defined by (2.1), (2.2) and the observation

$$
y(t)=C_{1} x(t)+C_{2} x(t-h)
$$

is given by

$$
\Xi(s)=\left(C_{1}+C_{2} e^{-h s}\right) \Delta(s)^{-1} B_{0} .
$$

We now give conditions under which the system (2.1)-(2.3) will be positive-real.

Lemma 2.1 Suppose that $\left(A_{0}, B_{0}\right)$ is controllable, $A_{0}+|\alpha| I$ is a stable semisimple matrix and $G_{0}(s)=C_{0}\left(s I-A_{0}\right)^{-1} B_{0}$ is positive-real. Then there exist matrices $L_{0}$, and $P=$ $P^{*} \geq 0$ such that

$$
\begin{aligned}
A_{0}^{*} P+P A_{0} & =-L_{0}^{*} L_{0} \\
B_{0}^{*} P & =C_{0}
\end{aligned}
$$

Moreover, $G(s)$ given by (2.8) is positive-real. 
Proof. (2.12), (2.13) follow from the finite-dimensional positive-real results. We rewrite (2.12) as follows

$$
\left(A_{0}+|\alpha| I\right)^{*} P+P\left(A_{0}+|\alpha| I\right)=-L_{0}^{*} L_{0}+2|\alpha| P .
$$

Denoting $K=L_{0}^{*} L_{0}-2|\alpha| P$ and $A_{1}=A_{0}+|\alpha| I$, we deduce the following from (2.14)

$$
\frac{d}{d t}\left\langle e^{A_{1} t} P x, e^{A_{1} t} P x\right\rangle=-\left\langle K e^{A_{1} t} P x, e^{A_{1} t} P x\right\rangle
$$

and since $A_{1}$ is a stable matrix

$$
\langle P x, x\rangle=\int_{0}^{\infty}\left\langle K e^{A_{1} t} P x, e^{A_{1} t} P x\right\rangle d t .
$$

Now $A_{1}$ is semisimple and so

$$
e^{A_{1} t} x=\sum_{i=1}^{n} e^{\lambda_{i} t}\left\langle x, e_{i}\right\rangle e_{i}
$$

where $\left(\lambda_{i}, e_{i}\right)$ are the eigenvalue-eigenvector pairs of $A_{1}$. Thus

$$
\begin{aligned}
\left\langle P e_{i}, e_{i}\right\rangle & =\int_{0}^{\infty} e^{2 \operatorname{Re} \lambda_{i} t}\left\langle K e_{i}, e_{i}\right\rangle d t \\
& =-\frac{1}{2 \operatorname{Re} \lambda_{i}}\left\langle K e_{i}, e_{i}\right\rangle
\end{aligned}
$$

and $\operatorname{Re} \lambda_{i}<0$ since $A_{1}$ is stable, and hence

$$
K=L_{0}^{*} L_{0}-2|\alpha| P \geq 0 .
$$

An easy calculation gives

$$
\begin{aligned}
\Pi(i \omega) & \\
\quad & G(i \omega)^{*}+G(i \omega) \\
& =B_{0}^{*}\left(-i \omega-A_{0}^{*}-\alpha e^{i \omega h}\right)^{-1}\left(L_{0}^{*} L_{0}-2 \alpha P \cos \omega h\right)\left(i \omega-A_{0}-\alpha e^{-i \omega h}\right)^{-1} B_{0} \\
& \geq B_{0}^{*}\left(-i \omega-A_{0}^{*}-\alpha e^{i \omega h}\right)^{-1}\left(L_{0}^{*} L_{0}-2|\alpha| P\right)\left(i \omega-A_{0}-\alpha e^{-i \omega h}\right)^{-1} B_{0} \\
& \geq 0,
\end{aligned}
$$

where we have used (2.12)-(2.14). Finally, $A$ is stable if $A_{0}+|\alpha| I$ is. $\square$

Next we obtain a spectral factorization for $\Pi$ of the form

$$
\Pi(i \omega)=\Xi(i \omega)^{*} \Xi(i \omega) .
$$

Lemma 2.2 There exist square matrices $C_{1}, C_{2} \in \mathcal{L}\left(\mathbb{C}^{n}\right)$ such that (2.15) holds with the spectral factor $\Xi$ given by (2.11). The matrices are any solutions of

$$
C_{1}^{*} C_{1}+C_{2}^{*} C_{2}=C_{0}^{*} C_{0} ; C_{1}^{*} C_{2}=C_{2}^{*} C_{1}=-2 \alpha P .
$$

Proof. If there exist $C_{1}, C_{2}$ satisfying (2.16) it is straightforward to verify that $\boldsymbol{\Xi}$ satisfies (2.15). So it remains to show the existence of $C_{1}, C_{2}$. Without loss of generality we can take $C_{1}, C_{2}$ to be symmetric and $\alpha$ to be positive. We choose a square matrix $F$ such that

$$
\left(C_{1}+C_{2}\right)^{*}\left(C_{1}+C_{2}\right)=C_{0}^{*} C_{0}-2 \alpha P=F^{*} F .
$$

Then, substituting $C_{2}=F-C_{1}$ we obtain the Riccati equation for $C_{1}$ :

$$
C_{1} F+F^{*} C_{1}-2 C_{1} C_{1}+4 \alpha P=0 .
$$


This has a solution which we take to be $C_{1}$ and $C_{2}=F-C_{1}$.

While in finite dimensions the existence of a spectral factor and controllability is sufficient to ensure the existence of a solution to the constrained Lyapunov equation, the infinite-dimensional case is more subtle. We refer to Curtain [1], section 7, for the rest of the proof of the existence to a solution to (2.12), (2.13). Essentially, it amounts to showing that the observation (2.9) is well-posed and this is known to be the case. So we may conclude that Theorem 1.2 holds under the assumptions

- $\left(A_{0}, B_{0}\right)$ is controllable

- $A_{0}+|\alpha| I$ is a stable matrix

- $G_{0}(s)=C_{0}\left(s I-A_{0}\right)^{-1} B_{0}$ is positive-real.

\section{Examples and Numerical Results}

We used the following matrices in (2.1), (2.3) to simulate the proposed adaptive scheme

$$
A_{0}=\left[\begin{array}{rr}
-1.0 & 0.4 \\
0.2 & -2.0
\end{array}\right], B_{0}=\left[\begin{array}{ll}
1.00 & 0.01 \\
0.01 & 1.00
\end{array}\right], C_{0}=\left[\begin{array}{ll}
2.01 & 1.04 \\
1.02 & 4.01
\end{array}\right] .
$$

The pair $\left(A_{0}, B_{0}\right)$ is indeed controllable and the solution to the constrained Lyapunov equation is

$$
P=\left[\begin{array}{ll}
2 & 1 \\
1 & 4
\end{array}\right] \text { with } L_{0}^{*} L_{0}=\left[\begin{array}{rr}
3.6 & 1.4 \\
1.4 & 15.2
\end{array}\right]
$$

One can easily check that $B_{0}^{*} P=C$ and thus $C_{0}\left(s I-A_{0}\right)^{-1} B$ is positive real. The scalar $\alpha$ is chosen as $\alpha=0.01$ with a delay $h=1$. In this case we have that $\operatorname{eig}\left(A_{0}+|\alpha| I\right)=$ $(-0.8255,-1.9745)$ which gives a stable $A_{0}+|\alpha| I$ matrix. Therefore all the conditions required for Theorem 1.2 to hold are satisfied.

The unknown parameter in the perturbation term is taken to be

$$
\Gamma=\left[\begin{array}{ll}
\Gamma_{11} & \Gamma_{12} \\
\Gamma_{21} & \Gamma_{22}
\end{array}\right]=\left[\begin{array}{cc}
4.0 \times 10^{-1} & 1.0 \times 10^{-4} \\
2.0 \times 10^{-4} & 2.0 \times 10^{-1}
\end{array}\right]
$$

The initial conditions for the state $x$, its estimate $\widehat{x}$ and the parameter $\widehat{\Gamma}(t)$ are given by

$$
x(\theta)=\left[\begin{array}{c}
\sin (4 \theta-1)+\sin (1) \\
\cos (4 \theta-1)
\end{array}\right], \widehat{x}(\theta)=\left[\begin{array}{c}
0.6(\sin (4 \theta-1)+\sin (1)) \\
1.4(\cos (4 \theta-1))
\end{array}\right],
$$

where $\theta \in[-1,0]$ and

$$
\widehat{\Gamma}(0)=\left[\begin{array}{ll}
2.0 \times 10^{-1} & 0.5 \times 10^{-4} \\
1.5 \times 10^{-4} & 3.0 \times 10^{-1}
\end{array}\right] .
$$

The plant input for this set of simulations was taken to be

$$
u(t)=\left[\begin{array}{c}
\cos (5.0 \pi t)+\sin (7.0 \pi t)+\sin (24.2 \pi t) \\
\sin (5.0 \pi t)+\sin (8.0 \pi t)+\cos (29.2 \pi t)
\end{array}\right] .
$$

The adaptive laws for the entries of the gain matrix become

$$
\begin{aligned}
\dot{\hat{\Gamma}}_{11}(t)=-5 \times 10^{-2} \varepsilon_{1}(t) y_{1}(t), & & \dot{\hat{\Gamma}}_{12}(t)=-3 \times 10^{-5} \varepsilon_{1}(t) y_{2}(t), \\
\dot{\hat{\Gamma}}_{21}(t)=-5 \times 10^{-5} \varepsilon_{2}(t) y_{1}(t), & & \dot{\hat{\Gamma}}_{22}(t)=-5 \times 10^{-2} \varepsilon_{2}(t) y_{2}(t),
\end{aligned}
$$

where $\varepsilon_{i}(t)=y_{i}(t)-\widehat{y}_{i}(t), i=1,2$. 

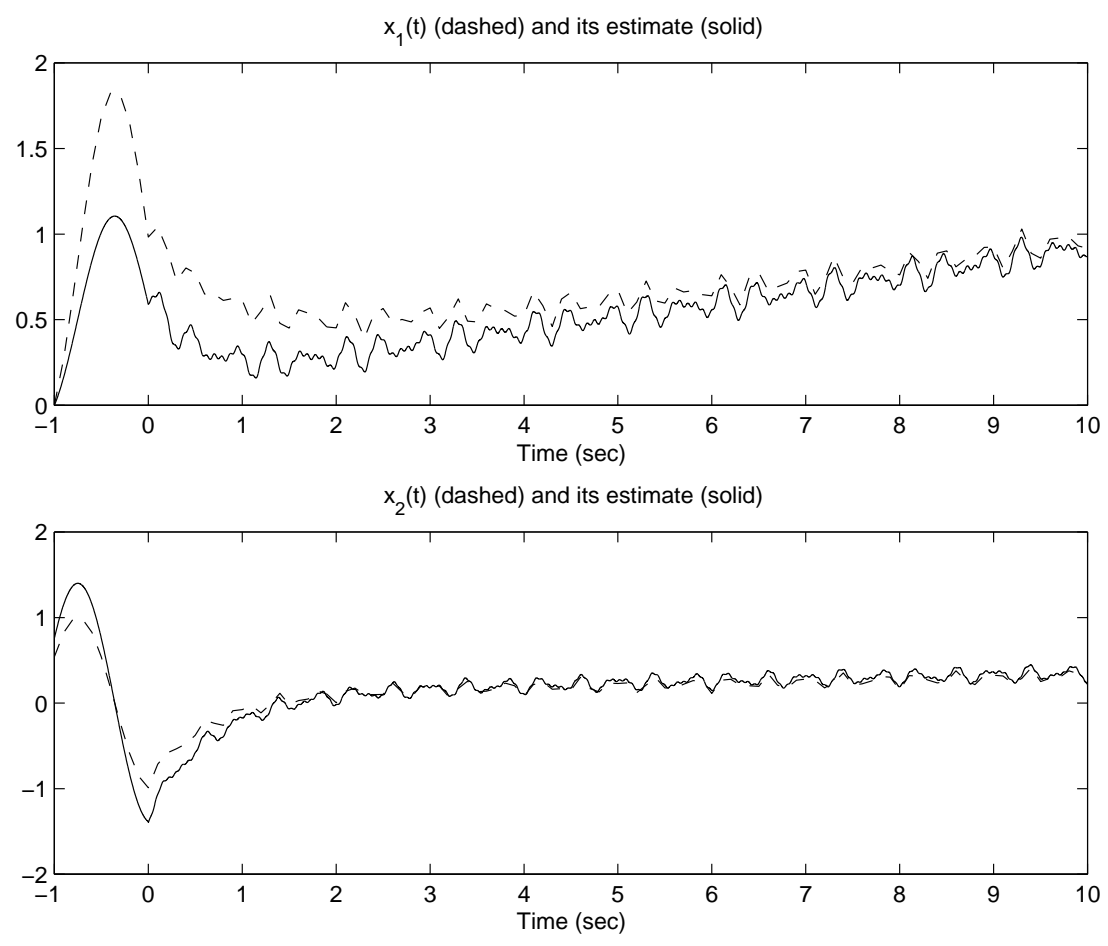

Figure 1: Evolution of (a) $x_{1}$ (dashed) and $\widehat{x}_{1}$ (solid), (b) $x_{2}$ (dashed) and $\widehat{x}_{2}$ (solid).

The above system was simulated using the approximation scheme developed in [4] with $N=12$ splines to discretize the interval $[-1,0]$, thus resulting in 26 equations for the state, 26 for its estimate and 4 for the parameter matrix $\Gamma$, totaling 56 equations. Due to the infinite dimensionality of the delay system, the discretization index $N=12$ was chosen as the smallest possible one that would provide a numerically reliable approximation to the above system. The resulting evolution system was integrated using the Fehlberg fourthfifth Runge-Kutta method based routine rkf45.f over the time interval $[0,30]$ sec. It should be noted that different combinations of the input signal $u(t)$ and initial conditions for the observer state were also tested which resulted in different convergence properties of the state and parameter errors. We present only the results with the above choice since they provided the required level of persistence of excitation needed for parameter convergence.

The evolution of the state components $x_{1}(t)$ and $x_{2}(t)$ is depicted in Figure 1 along with their estimates over the interval $[-1,10]$ seconds. Convergence of the observer state to the plant state can easily be concluded. The evolution of the parameter estimates along with their actual values are depicted in Figure 2. Parameter convergence is also achieved as is evident from the figure.

\section{References}

[1] R. F. CuRtain, Linear operator inequalities for stable weakly regular linear systems, manuscript, (1997).

[2] R. F. Curtain, M. A. Demetriou, And K. Ito, Adaptive compensators for perturbed positive real infinite dimensional systems, manuscript, (1997).

[3] R. F. Curtain and H. J. Zwart, An Introduction to Infinite Dimensional Linear Systems Theory, Texts in Applied Mathematics, Vol. 21, Springer-Verlag, Berlin, 1995.

[4] K. Ito AND F. KAPPEL, A uniformly differentiable approximation scheme for delay systems using splines, Applied Mathematics and Optimization, 23 (1991), pp. 217-262. 

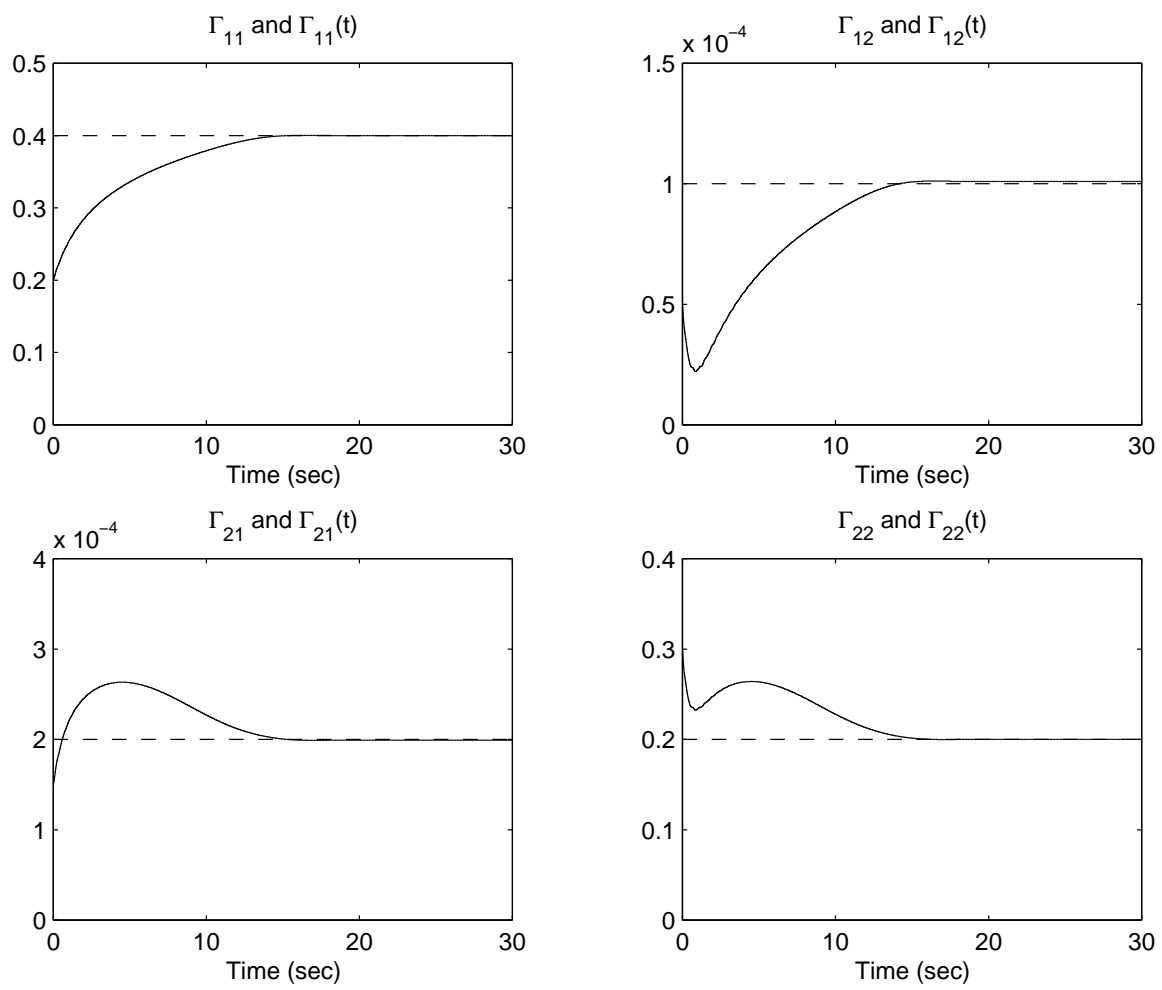

Figure 2: Evolution of the estimates $\widehat{\Gamma}_{i j}(t)$ (solid) and their actual values (dashed).

[5] H. K. KhaliL, Nonlinear Systems, Macmillan, New York, 1992.

[6] K. S. Narendra And A. M. Annaswamy, Stable Adaptive Systems, Prentice Hall, Englewood Cliffs, NJ, 1989. 